\title{
Modeling epidemics by the lattice Boltzmann method
}

DOI:

10.1103/PhysRevE.102.023301

\section{Document Version}

Accepted author manuscript

Link to publication record in Manchester Research Explorer

\section{Citation for published version (APA):}

De Rosis, A. (2020). Modeling epidemics by the lattice Boltzmann method. Physical Review E, 102(2), 023301. [023301]. https://doi.org/10.1103/PhysRevE.102.023301

\section{Published in:}

Physical Review E

\section{Citing this paper}

Please note that where the full-text provided on Manchester Research Explorer is the Author Accepted Manuscript or Proof version this may differ from the final Published version. If citing, it is advised that you check and use the publisher's definitive version.

\section{General rights}

Copyright and moral rights for the publications made accessible in the Research Explorer are retained by the authors and/or other copyright owners and it is a condition of accessing publications that users recognise and abide by the legal requirements associated with these rights.

\section{Takedown policy}

If you believe that this document breaches copyright please refer to the University of Manchester's Takedown Procedures [http://man.ac.uk/04Y6Bo] or contact uml.scholarlycommunications@manchester.ac.uk providing relevant details, so we can investigate your claim.

\section{OPEN ACCESS}




\title{
Modelling of epidemics by the lattice Boltzmann method
}

\author{
Alessandro De Rosis* \\ Department of Mechanical, Aerospace and Civil Engineering \\ The University of Manchester, Manchester, M13 9PL United Kingdom
}

(Dated: July 23, 2020)

\begin{abstract}
In this paper, we demonstrate that the lattice Boltzmann method can be successfully adopted to investigate the dynamics of epidemics. Numerical simulations prove the excellent accuracy properties of the approach that recovers the solution of the popular SIR model. Because spatial effects are naturally accounted for in the lattice Boltzmann formulation, the present scheme appears to be more competitive than traditional solution procedures. Interestingly, it allows us to simulate scenarios characterized by selective lockdown configurations.
\end{abstract}

PACS numbers: 47.11.-j, 82.40.Ck

Keywords: Lattice Boltzmann method, reaction-diffusion equation, epidemic

\section{MOTIVATION}

During its history, the mankind has seen the rise, ${ }^{50}$ spread and outbreak of a rich variety of infectious dis- ${ }^{51}$ eases which have affected a significant portion of pop- ${ }^{52}$ ulation. The first ever registered case is the Plague of ${ }^{53}$ Athens, a typhoid fever that has killed nearly $100000^{54}$ people in Greece around 430 B.C. [1]. Diseases in- ${ }^{55}$ duced by the Yersinia pestis bacterium, Variola virus ${ }^{56}$ and zoonotic viruses (as the swine and avian flu) are ${ }^{57}$ among the most famous and dramatic epidemics that ${ }^{58}$ have appeared through the centuries.

A deep understanding of the process leading to the ${ }^{60}$ spread of a disease is instrumental to contain, delay and ${ }^{61}$ mitigate its potential outbreak and it is also helpful to ${ }^{62}$ evaluate strategies to control an epidemic $[2,3]$. The ${ }^{63}$ first empirical quantitative study of human deaths and ${ }^{64}$ diseases has been carried out by Graunt in 1662 [4], who ${ }^{65}$ discussed demographic problems in Britain and listed the ${ }^{66}$ number and causes of deaths of London parishes. After ${ }^{67}$ a century, Bernoulli provided a deterministic model to ${ }^{68}$ defend the practice of inoculating against smallpox [5].

In 1927, a seminal contribution to the modelling of 70 epidemics has been proposed by Kermack et al. [6], who ${ }^{71}$ introduced a simple yet effective compartmental model. ${ }^{72}$ Specifically, a certain population of fixed size is divided ${ }^{73}$ into three groups: susceptibles $(\mathcal{S})$, who can get the dis- ${ }^{74}$ eases; infected $(\mathcal{I})$, who have the disease; and recovered ${ }^{75}$ $(\mathcal{R})$, who were infected and then have become immune. ${ }^{76}$ Nowadays, their so-called SIR model represents the most consolidated approach to predict the time evolution of a 77 disease. It consists of solving three equations:

$$
\begin{aligned}
\frac{\partial \mathcal{S}}{\partial t} & =-\frac{\beta \mathcal{S I}}{N}, \\
\frac{\partial \mathcal{I}}{\partial t} & =\frac{\beta \mathcal{S I}}{N}-\gamma \mathcal{I}, \\
\frac{\partial \mathcal{R}}{\partial t} & =\gamma \mathcal{I},
\end{aligned}
$$$$
82
$$

$$
84
$$

where $t$ is the time, $N=\mathcal{S}+\mathcal{I}+\mathcal{R}$ is the total population, positive constants $\beta$ and $\gamma$ are the contact and recovery rates, respectively. Consistently, it is possible to define the famous reproduction number $R_{0}=\beta / \gamma$. The original formulation provided by the SIR model can be further enriched by accounting for maternally-derived immunity, vaccinations, exposition and incubation times, among the others.

One of the major assumptions behind Eqs. (1) is that environmental conditions are considered homogeneous. However, individual organisms typically interact with the surrounding physical environment and other organisms. Climate and chemical composition, as well as other environmental factors, can vary from a place to another and can affect the dynamics of populations and communities. Therefore, spatial effects can play an important role in the spread of epidemics. Notably, Mollison [7] investigated spatial models for epidemic spread. It should be noted that this paper deals with spatial effects and not spatial epidemiology. The latter term is nowadays used to describe the geographic variation of disease incidence in relation to demographic or socio-economic factors, with time scales much larger than the ones associated with the propagation of infectious diseases [8]. Building on the pioneering work by Turing [9], many studies addressed the importance of spatial effects, showing how population diffusion impacts the formation of spatial patterns [10-17].

Here, it is suggested to account for spatial effects by modifying Eqs. (1) as follows:

$$
\begin{aligned}
\frac{\partial \mathcal{S}}{\partial t} & =-\frac{\beta \mathcal{S} \mathcal{I}}{N}+d^{\mathcal{S}} \nabla^{2} \mathcal{S}, \\
\frac{\partial \mathcal{I}}{\partial t} & =\frac{\beta \mathcal{S} \mathcal{I}}{N}-\gamma \mathcal{I}+d^{\mathcal{I}} \nabla^{2} \mathcal{I}, \\
\frac{\partial \mathcal{R}}{\partial t} & =\gamma \mathcal{I}+d^{\mathcal{R}} \nabla^{2} \mathcal{R},
\end{aligned}
$$

where $\mathcal{S}=\mathcal{S}(\mathbf{x}, t), \mathcal{I}=\mathcal{I}(\mathbf{x}, t), \mathcal{R}=\mathcal{R}(\mathbf{x}, t), \mathbf{x}=[x, y]$ being the spatial coordinate in two dimensions. Moreover, $d^{\mathcal{S}}, d^{\mathcal{I}}$ and $d^{\mathcal{R}}$ are the diffusion coefficients of populations $\mathcal{S}, \mathcal{I}$ and $\mathcal{R}$, respectively, and $\nabla^{2}=\frac{\partial^{2}}{\partial x^{2}}+\frac{\partial^{2}}{\partial y^{2}}$ is the Laplacian operator. From a biological and be- 
havioural perspective, the diffusion of individuals can be connected to several aspects, such as food/medicine hunting or leaving zones with high infection risks. From a mathematical viewpoint, Eqs. (2) represent a set of three reaction-diffusion equations, where the last term of each right-hand side is the diffusive part and the remaining terms at the same side account for reaction processes. It should be noted that the solution of Eqs. (2) requires the estimation of second-order derivative by finite differences that can involve a non-negligible amount of computational time.

Interestingly, Ponce Dawson et al. [18] showed that an robust alternative to solve a reaction-diffusion equation is represented by the lattice Boltzmann method (LBM) $\left[19-{ }^{125}\right.$ $21]$. The aim of this paper is to propose, test and validate an LB formulation that can be successfully employed to perform accurate simulations of the dynamics of epi- ${ }^{127}$ demics. In SEC. II, the adopted methodology is outlined and accompanied by a Chapman-Enskog expansion and ${ }_{128}$ a linear stability analysis. Its accuracy is confirmed by numerical results in SEC. III, where the capability to129 simulate a selective lockdown in an urban scenario is also shown. Eventually, some concluding remarks are given in ${ }^{130}$ SEC. IV.

\section{METHODOLOGY}

In this section, the LB scheme to simulate the spread ${ }^{135}$ ing of epidemics is presented. The Chapman-Enskog ${ }^{136}$ expansion demonstrates that our methodology recovers ${ }^{137}$ Eqs. (2). Eventually, a von Neumann linear stability analysis shows that the stability of the algorithm deteriorates for vanishing values of the diffusivity and high ${ }^{130}$ values of the contact and recovery rates.

\section{A. Lattice Boltzmann method for epidemics}

The governing lattice Boltzmann equation (LBE) predicts the space-time evolution of the particle distribution functions $f_{i}^{k}$ colliding and streaming along the links $\mathbf{c}_{i}=\left[c_{i x}, c_{i y}\right]$ of the D2Q9 Cartesian lattice, where $i=0, \ldots, 8, k=\mathcal{S}, \mathcal{I}, \mathcal{R}$ and

$$
\begin{aligned}
& c_{i x}=[0,-1,-1,-1,0,1,1,1,0], \\
& c_{i y}=[0,1,0,-1,-1,-1,0,1,1] .
\end{aligned}
$$

The LBEs read as follows:

$$
f_{i}^{k}\left(\mathbf{x}+\mathbf{c}_{i}, t+1\right)=f_{i}^{k}(\mathbf{x}, t)+\Omega_{i, \mathrm{NR}}^{k}(\mathbf{x}, t)+\Omega_{i, \mathrm{R}}^{k}(\mathbf{x}, t),
$$

where the non-reactive (NR) parts obey the BGK approximation [22], that is

$$
\Omega_{i, \mathrm{NR}}^{k}=\frac{1}{\tau^{k}}\left(f_{i, e q}^{k}-f_{i}^{k}\right) .
$$

Particle distributions relax to an equilibrium state defined as $[23,24]$

$$
f_{i, e q}^{k}=w_{i} \rho^{k}
$$

where

$$
\rho^{k}=\sum_{i} f_{i}^{k}
$$

is the density of population $k$. The weights associated to the D2Q9 lattice [25] are

$$
w_{i}=[4 / 9,1 / 36,1 / 9,1 / 36,1 / 9,1 / 36,1 / 9,1 / 36,1 / 9]
$$

and the relaxation time is

$$
\tau^{k}=3 d^{k}+\frac{1}{2} .
$$

Depending on the considered group, the reactive $(\mathrm{R})$ parts of the LBEs assume different expressions, i.e.

$$
\begin{aligned}
& \Omega_{i, \mathrm{R}}^{\mathcal{S}}=w_{i}\left(-\frac{\beta \rho^{\mathcal{S}} \rho^{\mathcal{I}}}{\rho^{N}}\right), \\
& \Omega_{i, \mathrm{R}}^{\mathcal{I}}=w_{i}\left(\frac{\beta \rho^{\mathcal{S}} \rho^{\mathcal{I}}}{\rho^{N}}-\gamma \rho^{\mathcal{I}}\right), \\
& \Omega_{i, \mathrm{R}}^{\mathcal{R}}=w_{i} \gamma \rho^{\mathcal{I}},
\end{aligned}
$$

with $\rho^{N}=\sum_{k} \rho^{k}$.

Two advantages of the LBM can be immediately appreciated: (i) the reactive part is simply introduced by adding an external source term projected through the weights into the distributions space, and (ii) the diffusion term in Eqs. (2) is directly and naturally accounted for in the non-reactive part (i.e., the collision) without requiring the computation of any spatial derivatives.

\section{B. Chapman-Enskog expansion}

We provide a formal proof that the LBEs in Eqs. (4) recover the SIR equations in Eqs. (2) by performing the Chapman-Enskog expansion. To this end, let us rewrite Eq. (4) as

$$
\begin{aligned}
f_{i}^{k}\left(\mathbf{x}+\varepsilon \mathbf{c}_{i}, t+\varepsilon\right)= & f_{i}^{k}(\mathbf{x}, t)+\frac{1}{\tau^{k}}\left[f_{i, e q}^{k}(\mathbf{x}, t)-f_{i}^{k}(\mathbf{x}, t)\right] \\
& +\Omega_{i, \mathrm{R}}^{k}(\mathbf{x}, t),
\end{aligned}
$$

where $\varepsilon$ is a small parameter. Using the Taylor expansion, it can be rewritten as

$f_{i}^{k}\left(\mathbf{x}+\varepsilon \mathbf{c}_{i}, t+\varepsilon\right)-f_{i}^{k}(\mathbf{x}, t)=\sum_{n} \frac{\varepsilon^{n}}{n !}\left(\frac{\partial}{\partial t}+\mathbf{c}_{i} \frac{\partial}{\partial \mathbf{x}}\right)^{n} f_{i}^{k}(\mathbf{x}, t)$.

It is also assumed that

$$
f_{i}^{k}=\sum_{n} \varepsilon^{n} f_{i}^{(k, n)}
$$

where $f_{i}^{(k, 0)}=f_{i, e q}^{k}$. Changes at different time scales are discussed by introducing $t_{n}=\varepsilon^{n} t$ and

$$
\frac{\partial}{\partial t}=\sum_{n} \varepsilon^{n} \frac{\partial}{\partial t_{n}} .
$$


Indeed, the equation of order $\varepsilon$ is

$$
\Delta f_{i}^{(k, 0)}=-\frac{1}{\tau^{k}} f_{i}^{(k, 1)}+\Omega_{i, R}^{(k, 1)},
$$

where $\Delta=\frac{\partial}{\partial t_{0}}+\mathbf{c}_{i} \frac{\partial}{\partial \mathbf{x}}$.

The equation of order $\varepsilon^{2}$ is

$\frac{\partial}{\partial t_{1}} f_{i}^{(k, 0)}+C_{2} \Delta^{2} f_{i}^{(k, 0)}+\tau^{k} \Delta \Omega_{i, R}^{(k, 1)}=-\frac{1}{\tau^{k}} f_{i}^{(k, 2)}+\Omega_{i, R}^{(k, 2)}$.

Under the conditions

$$
\sum_{i} f_{i, e q}^{k}=\sum_{i} f_{i}^{k}, \quad \sum_{i} f_{i}^{(k, 0)} \mathbf{c}_{i}=0
$$

let us take a summation over $i$ of Eq. (15), that results in

$$
\frac{\partial}{\partial t_{0}} \rho^{k}=\sum_{i} \Omega_{i, R}^{(k, 1)}=R^{(k, 1)},
$$

where $R^{(k, 1)}$ is the reaction term at the right-hand sides of Eqs. (2) and

$$
\Omega_{i, R}^{(k, 1)}=w_{i} R^{(k, 1)} .
$$

By taking the summation over $i$ of Eq. (16), we have

$$
\frac{\partial}{\partial t_{1}} \rho^{k}+C_{2} \sum_{i} \Delta^{2} f_{i}^{(k, 0)}+\sum_{i} \tau \Delta \Omega_{i, R}^{(k, 1)}=\sum_{i} \Omega_{i, R}^{(k, 2)} .
$$

Now, let us write

$$
\pi_{l, m}^{(k, 0)}=\sum_{i} f_{i}^{(k, 0)} c_{i l} c_{i m}=\lambda^{k} \delta_{l, m} \rho^{k},
$$

where $l$ and $m$ span the Eulerian basis, $\delta_{l, m}$ is the Kronecker delta and

$$
\lambda^{k}=\frac{d^{k}}{\varepsilon(\tau-1 / 2)} .
$$

Eq. (20) becomes

$$
\frac{\partial}{\partial t_{1}} \rho^{k}+C_{2} \lambda^{k} \nabla^{2} \rho^{k}=0
$$

Therefore, taking (15) $+(16) \times \varepsilon$ and summing over $i$ allow us to write

$$
\frac{\partial}{\partial t} \rho^{k}+\varepsilon C_{2} \frac{\partial^{2}}{\partial x_{l} \partial x_{m}} \pi_{l, m}^{(k, 0)}=\sum_{i} \Omega_{i, R}^{(k, 1)},
$$

that becomes

$$
\frac{\partial}{\partial t} \rho^{k}=R^{(k, 1)}+d^{k} \nabla^{2} \rho^{k} .
$$

One can immediately appreciate the equivalence between Eq. (25) and any of Eqs. (2).

\section{Linear stability analysis}

Here, the results of a von Neumann linear stability analysis are presented. We notice that many efforts have been devoted to investigate the stability of the sole collision operator in the case of LB schemes able to recover the Navier-Stokes equations [26-32]. Interestingly, few works $[33,34]$ show a linear stability analysis when a force (source) term is considered. Here, we need to account for both the collision operator and the source term, that are the non-reactive and reactive parts of the governing LB equation, respectively.

For simplicity, let us consider just the equation for the evolution of the recovered people. To lighten the notation, the superscript $\mathcal{R}$ will be implicitly assumed in the rest of this section. Therefore, we can say

$$
f_{i}\left(\mathbf{x}+\mathbf{c}_{i}, t+1\right)=f_{i}(\mathbf{x}, t)+\frac{1}{\tau}\left(f_{i, e q}-f_{i}\right)+w_{i} \gamma \rho,
$$

that can be rewritten as

$$
\begin{aligned}
f_{i}\left(\mathbf{x}+\mathbf{c}_{i}, t+1\right)= & \left(1-\frac{1}{\tau}\right) f_{i}(\mathbf{f}(\mathbf{x}, t))+\frac{1}{\tau} f_{i, e q}(\mathbf{f}(\mathbf{x}, t)) \\
& +S_{i}(\mathbf{f}(\mathbf{x}, t)),
\end{aligned}
$$

where $\mathbf{f}=\left[f_{0}, \ldots, f_{8}\right]$ is a vector collecting the particle distribution functions and $S_{i}$ collects the source reactive term. Distributions can be rearranged as

$$
f_{i}(\mathbf{x}, t)=f_{i}^{\prime}+\delta f_{i}(\mathbf{x}, t),
$$

where $f_{i}^{\prime}=f_{i, e q}(\rho=1)$ is an unperturbed solution of Eq. (27) and $\delta f_{i}(\mathbf{x}, t)$ are small perturbations. Linearization takes place as follows [33, 34]:

$$
\begin{gathered}
f_{i, e q}(\mathbf{f})=f_{i, e q}\left(\mathbf{f}^{\prime}+\delta \mathbf{f}\right) \approx f_{i, e q}\left(\mathbf{f}^{\prime}\right)+\sum_{s=0}^{8} \frac{\partial f_{i, e q}}{\partial f_{s}}\left(\mathbf{f}^{\prime}\right) \delta f_{s} \\
S_{i}(\mathbf{f})=S_{i}\left(\mathbf{f}^{\prime}+\delta \mathbf{f}\right) \approx S_{i}\left(\mathbf{f}^{\prime}\right)+\sum_{s=0}^{8} \frac{\partial S_{i}}{\partial f_{s}}\left(\mathbf{f}^{\prime}\right) \delta f_{s},
\end{gathered}
$$

where $\mathbf{f}^{\prime}=\left[f_{0}^{\prime}, \ldots, f_{8}^{\prime}\right]$. This allows us to write

$$
\begin{aligned}
S_{i}\left(\mathbf{x}+\mathbf{c}_{i}, t+1\right)= & \left(1-\frac{1}{\tau}\right) S_{i}(\mathbf{x}, t)+\frac{1}{\tau} \sum_{s=0}^{8} A_{i s} \delta f_{s}(\mathbf{x}, t) \\
& +\sum_{s=0}^{8} B_{i s} \delta f_{s}(\mathbf{x}, t),
\end{aligned}
$$

where

are Jacobi matrices. The solution of Eq. (30) can be

given as

$$
\begin{aligned}
& A_{i s}=\frac{\partial f_{i, e q}}{\partial f_{s}}=\frac{\partial f_{i, e q}}{\partial \rho} \frac{\partial \rho}{\partial f_{s}}, \\
& B_{i s}=\frac{\partial S_{i}}{\partial f_{s}}=\frac{\partial S_{i}}{\partial \rho} \frac{\partial \rho}{\partial f_{s}}
\end{aligned}
$$

$$
S_{i}(\mathbf{x}, t)=F_{i}(t) \exp (\iota \boldsymbol{\Theta} \cdot \mathbf{x}),
$$


where $\iota$ is the imaginary unit, $\boldsymbol{\Theta}=\left(\theta_{x}, \theta_{y}\right)$ with $\theta_{x, y} \in_{178}$ $[-\pi, \pi]$. The following system is obtained

$$
F_{i}(t+1)=\sum_{s=0}^{8} G_{i s} F_{s}(t)
$$

where $G_{i s}$ are the component of a transition matrix $\mathbf{G}_{184}$ defined as [33, 34]

$G_{i s}=\left[\left(1-\frac{1}{\tau}\right)+\frac{1}{\tau} A_{i s}+B_{i s}\right] \exp \left(\iota \Theta \cdot \mathbf{c}_{i}\right), \quad$ if $i=s,{ }_{188}{ }_{187}$ $G_{i s}=\left[\frac{1}{\tau} A_{i s}+B_{i s}\right] \exp \left(\iota \boldsymbol{\Theta} \cdot \mathbf{c}_{i}\right), \quad$ if $i \neq s$.

Hence, the solution is stable if the maximum complex ${ }^{191}$ modulus of the eigenvalues of $\mathbf{G}$ is smaller than 1 . By ${ }^{192}$ varying $\gamma \in[0: 2]$ and $\tau \in[0.5: 2]$, we compute this ${ }_{193}$ quantity by the QR-algorithm and it is plotted in FIG. $1_{194}$ Some considerations should be drawn. Independently ${ }_{195}$

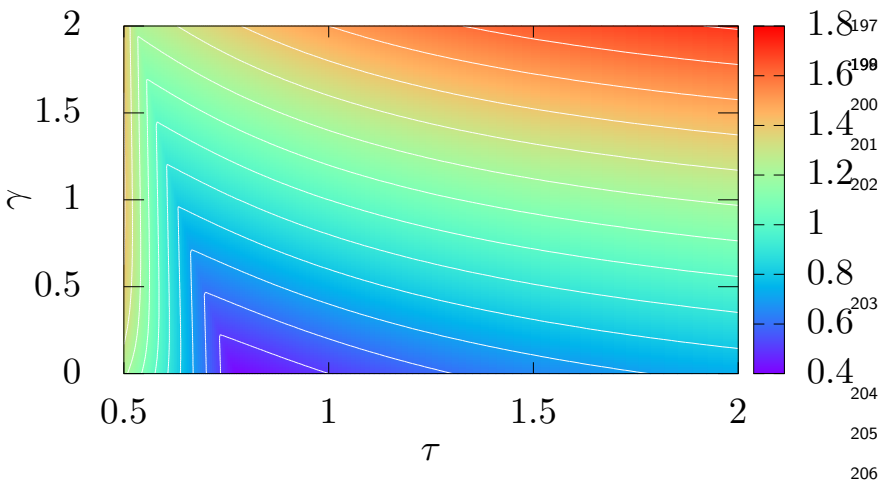

FIG. 1. Linear stability analysis: Map of the maximum com- ${ }^{207}$ plex modulus of the eigenvalues of $\mathbf{G}$.

from the value of the recovery rate $\gamma$, the solution be- ${ }_{211}$ comes unstable if $\tau \rightarrow 0.5$ (i.e., in the limit of vanishing ${ }_{212}$ diffusivity). The optimal zone is found when $0.7 \leq \tau \leq 1_{213}$ and $\gamma \leq 1$, where minima of the complex modulus are $_{214}$ localized. Progressively larger maxima of the complex ${ }_{215}$ modulus arise as $\tau$ and $\gamma$ grow, with the latter having $\mathrm{a}_{216}$ more prominent deleterious effect. For the sake of com- ${ }_{217}$ pleteness, some of the values shown in FIG. 1 are reported ${ }_{218}$ in TABLE I too.

\section{RESULTS AND DISCUSSION}

In this Section, we first demonstrate that the devised 226 approach is consistent with the classical SIR model. Sec-227 ondly, the effect of the diffusion in the spreading of epi-228 demics is discussed. Finally, an urban scenario repre-229 sentative of Midtown Manhattan is investigated with a230 selective lockdown configuration.

\section{A. Recovery of the SIR model}

The accuracy and reliability of the present method is assessed by comparing the results from LB simulations to the predictions obtained by the solution of Eqs. (1). In the latter, a population of $N=40000$ individuals is assumed where a certain fraction $\mathcal{I}(t=0)=$ $0.1 \%, 1 \%, 10 \%$ is initially infected. Moreover, the recovery rate is set to $\gamma=5$ days $^{-1}$ and the reproduction number is varied as $R_{0}=1.5,3,5$. In order to convert the problem to the LB world, a square domain is considered where each side has length $\sqrt{N}=200$. Then, the system is initialized as follows:

$$
\begin{aligned}
\rho^{\mathcal{I}}(t=0) & =\mathcal{I}(t=0), \\
\rho^{\mathcal{S}}(t=0) & =1-\mathcal{I}(t=0), \\
\rho^{\mathcal{R}}(t=0) & =0 .
\end{aligned}
$$

Since the original SIR model is diffusion-free, to achieve the same scenario the diffusion coefficient should be set to low values, i.e. $d^{\mathcal{S}}=d^{\mathcal{I}}=d^{\mathcal{R}}=10^{-5}$.

In FIG. 2, the time evolution of the fraction of infected people (also known as epidemic curve) is plotted for the aforementioned values of $R_{0}$ and $\mathcal{I}(t=0)$. One can immediately appreciate the excellent agreement between findings from the two approaches, with a maximum relative discrepancy of $\sim 0.02 \%$ [35].

\section{B. Effect of the diffusivity}

Here, the role of the diffusion on the dynamics of epidemics is elucidated. Let us first assume a common value for all the diffusion coefficients, i.e. $d^{\mathcal{S}}=d^{\mathcal{I}}=d^{\mathcal{R}}=d$. Let us consider the same square domain of dimensions $200 \times 200$ as before. At the beginning of the simulations, the fraction of infected people occupies a small circular region of radius $r=20$ with its center located at $\left(x_{c}, y_{c}\right)=(100,100)$, while the rest of the domain is composed of susceptible persons. In other words, $\rho^{\mathcal{I}}(t=0)=$ 1 and $\rho^{\mathcal{S}}(t=0)=0$ if $\left(x-x_{c}\right)^{2}+\left(y-y_{c}^{2}\right)<r^{2}$, otherwise $\rho^{\mathcal{I}}(t=0)=0$ and $\rho^{\mathcal{S}}(t=0)=1$. It corresponds to have the $\sim 3.14 \%$ of the population initially infected. The reproduction number $R_{0}$ varies as before and three values of $d$ are used, i.e. $d=0.0005,0.001,0.01$. In FIG. 3, the epidemic curves are drawn for all the combinations of $R_{0}$ and $d$. We observe that, as the diffusion increases, the peak of the infection grows and appears progressively earlier. Indeed, the diffusion (movement) of individuals promotes and accelerate the spread of the disease. Our results corroborate the observations that isolation and social distancing are a good measure to contain, delay and mitigate the spread of an infection.

Eventually, the dynamics of epidemics when groups diffuse/move differently is dissected. By setting $R_{0}=5$, two configurations are investigated. In the former, the diffusion coefficient associated to infected individuals is kept fixed to $d^{\mathcal{I}}=0.001$, while the other two assume the 


\begin{tabular}{c|c|c|c|c|c|c|c}
\hline \hline$\gamma \tau$ & 0.50 & 0.75 & 1.00 & 1.25 & 1.50 & 1.75 & 2.00 \\
\hline 0.00 & 1.3693 & 0.4336 & 0.5000 & 0.5850 & 0.6479 & 0.6950 & 0.7312 \\
\hline 0.25 & 1.4090 & 0.5184 & 0.6250 & 0.7087 & 0.7699 & 0.8156 & 0.8509 \\
\hline 0.50 & 1.4124 & 0.6389 & 0.7500 & 0.8328 & 0.8928 & 0.9375 & 0.9718 \\
\hline 0.75 & 1.3973 & 0.7609 & 0.8750 & 0.9572 & 1.0162 & 1.0600 & 1.0937 \\
\hline 1.00 & 1.3758 & 0.8837 & 1.0000 & 1.0817 & 1.1399 & 1.1831 & 1.2162 \\
\hline 1.25 & 1.3539 & 1.0070 & 1.1250 & 1.2063 & 1.2639 & 1.3065 & 1.3392 \\
\hline 1.50 & 1.3339 & 1.1307 & 1.2500 & 1.3309 & 1.3881 & 1.4302 & 1.4625 \\
\hline 1.75 & 1.3162 & 1.2547 & 1.3750 & 1.4557 & 1.5124 & 1.5541 & 1.5861 \\
\hline 2.00 & 1.3009 & 1.3789 & 1.5000 & 1.5804 & 1.6368 & 1.6782 & 1.7099 \\
\hline \hline
\end{tabular}

TABLE I. Linear stability analysis: Maximum complex modulus of the eigenvalues of $\mathbf{G}$ for different combinations of $\tau$ and $\gamma$.

same value that varies as $d^{\mathcal{S}}=d^{\mathcal{R}}=0.0005,0.001,0.1 .276$ Making reference to FIG. 4, the spatio-temporal evolu-277 tion of the epidemic is substantially insensitive to changes 278 of the diffusion coefficient associated to susceptible ( $\operatorname{and}_{279}$ recovered) people. Interestingly, findings depicted in 280 FIG. 5 are considerably more appealing. Here, result\$281 from the latter configuration are reported, where $d^{\mathcal{S}}=282$ $d^{\mathcal{R}}$ is enforced to 0.001 , while the other coefficient varies283 as $d^{\mathcal{I}}=0.0005,0.001$ and 0.1 . The detrimental role284 played by the spread of infected individuals clearly stems.286 In fact, the peak of the epidemic curve assumes higher ${ }_{287}$ values and moves to an earlier time as $d^{\mathcal{I}}$ increases. This288 corroborates data in [17], where it has been found that289 the fraction of infected people increases along with the 290 increase of the corresponding diffusion coefficient. Based 292 on our observations, we can conclude that isolating in-293 fected individuals is more important than applying the 294 same action on healthy persons.

\section{Urban scenario with selective lockdown}

In the last numerical experiment, we focus on a very ${ }^{301}$ particular situation, that is the spreading of an epidemic ${ }^{302}$ in Midtown Manhattan. Specifically, a portion of Man-30з hattan is selected as it is bounded by the 23rd Street to the 59 Street vertically and by the Hudson and East Rivers horizontally. This corresponds to an area of $\sim^{304}$ $8.7 \mathrm{~km}^{2}$ that has a population density of $28000 \mathrm{ab} / \mathrm{km}^{2}$. The choice of this particular zone is inspired by the fact305 that its roads network reminds and fits the Cartesian $\mathrm{LB}_{306}$ lattice. $\gamma=5$ days $^{-1}$ and $R_{0}=2.5$ are set. At the begin-307 ning of the simulation, the $10 \%$ of the population of the 308 area is assumed to be infected and randomly distributed.309 An uniform diffusivity $d=0.01$ (in lattice units) is im-310 posed. The map of the infected people is depicted in 311 FIG. 6 for different days, with the peak shown at Day 312 17. Interestingly, the outlined methodology allows us to313 simulate scenarios characterized by a selective lockdown,314 where the diffusion/movement of people is reduced (or 315 even prevented) in a certain specific region of the compu-316 tational domain. To this end, we run a second simulation 317 with the same configuration as above, but we assign a dif-318 fusivity reduced by a factor 100 to the area corresponding ${ }_{319}$ to the Hell's Kitchen district. The resultant simulation is characterized by non-uniform values of the diffusivity. The Hell's Kitchen district area goes from the 34th Street to the 59 Street vertically, and from the Hudson River to the 8 th Avenue to the horizontally. FIG. 7 shows the map of infected people at different days. The important role played by the reduced diffusivity is clearly visible. Indeed, one can immediately appreciate that the spreading of the epidemic is considerably delayed and mitigated in the Hell's Kitchen district. This result is much more emphasized in FIG. 8, where the map of infected people is sketched in the two configurations at Day 12. The colour contrast manifests the corresponding very different density of infected people between Hell's Kitchen and the rest of Midtown Manhattan. The epidemic curve in the two configurations are reported in FIG. 9. We found that the adoption of a reduced diffusivity in a certain specific region leads to a global peak reduction of $\sim 13 \%$.

It should also be noted that the zero-diffusivity case is not sufficient to capture the lockdown physics. In fact, in a qualitatively perfect lockdown condition, people stop interacting with each other and the contact rate should also go to zero, while the present model keeps $\beta$ as a constant. The interested reader can refer to [36] for a more detailed discussion related to this aspect.

\section{CONCLUSIONS}

In this paper, we proposed a lattice Boltzmann method to model the dynamics of epidemics. The governing reaction-diffusion LB equations accurately recovers the solution of the popular SIR model. This has been numerically demonstrated by means of simulations and theoretically proved by the Chapman-Enskog expansion. The von Neumann linear stability analysis highlights the possible stability limits of the scheme. Given the intrinsic nature of the approach, spatial effects are directly and naturally accounted for without the need of computing any derivatives. The methodology results in a simple algorithmic procedure to successfully unravel the dynamics of epidemics and to study containment strategies, as a selective lockdown in an urban scenario (as shown). Intriguingly, the diffusivity can be linked to the mobil- 

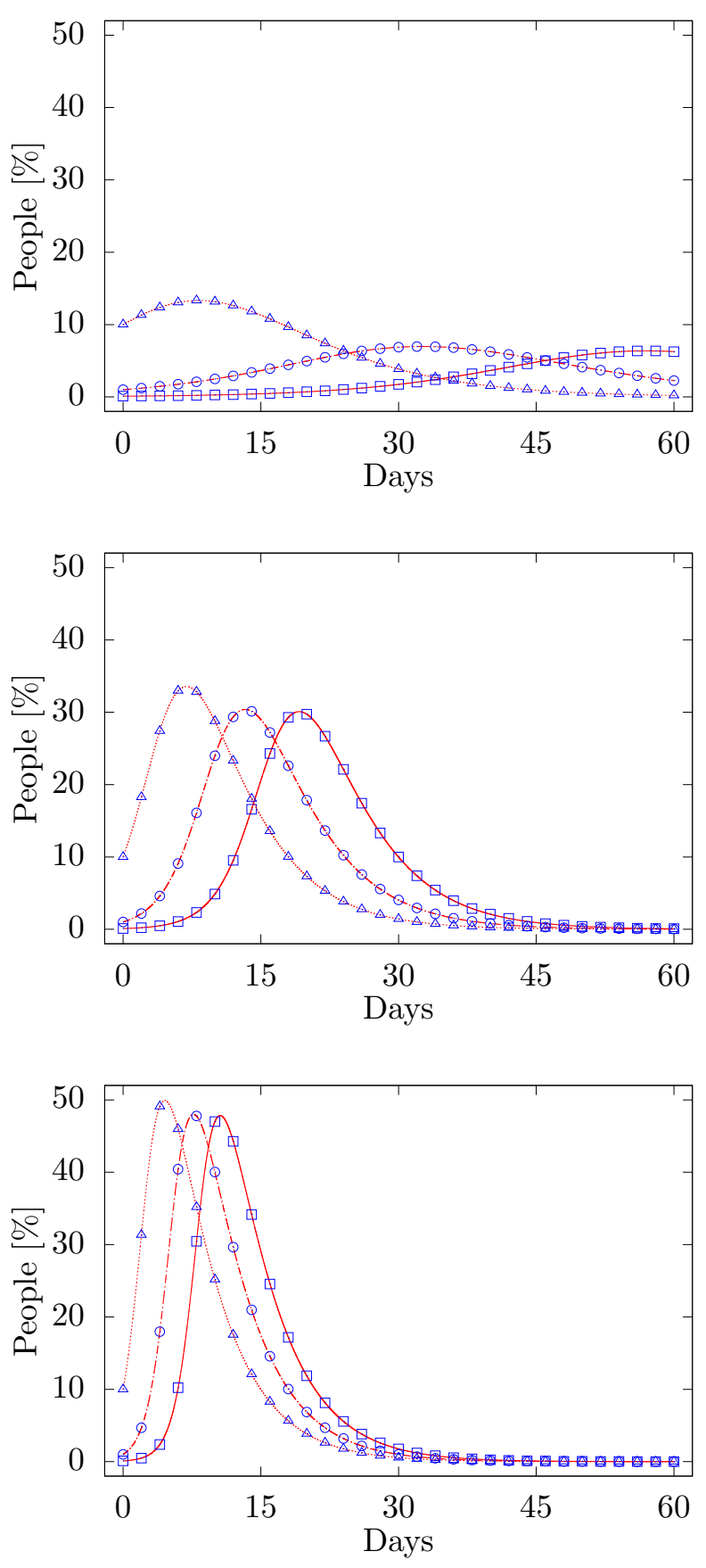

FIG. 2. Time evolution of the fraction of infected people for different values of $R_{0}$, i.e 1.5 (top), 3 (center) and 5 (bottom) and $\mathcal{I}(t=0)$, i.e. $0.1 \%$ (solid line and squares), $1 \%$ (dashdotted line and circles) and $10 \%$ (dotted line and triangles.) Lines and symbols correspond to results obtained by the SIR model (see Eqs. (1)) and the present LB one, respectively.

ity of a certain area, hence allowing us to simulate more realistic configurations.
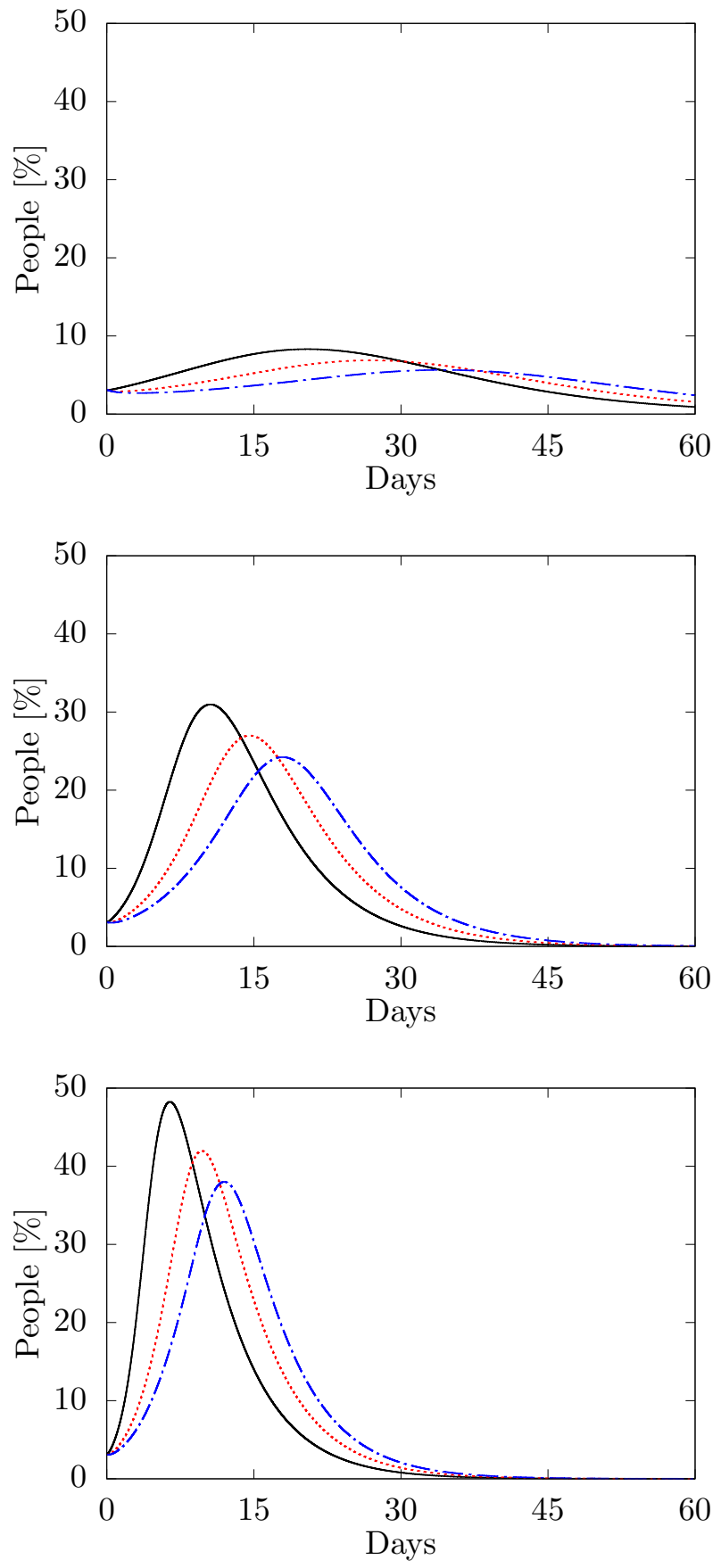

FIG. 3. Time evolution of the fraction of infected people for different values of $R_{0}$, i.e 1.5 (top), 3 (center) and 5 (bottom) and $d$, i.e. 0.0005 (blue dash-dotted line), 0.001 (red dotted) and 0.01 (black solid line.)

\section{ACKNOWLEDGEMENTS}

The author would like to thank Dr. C. Coreixas for valuable hints and suggestions. 


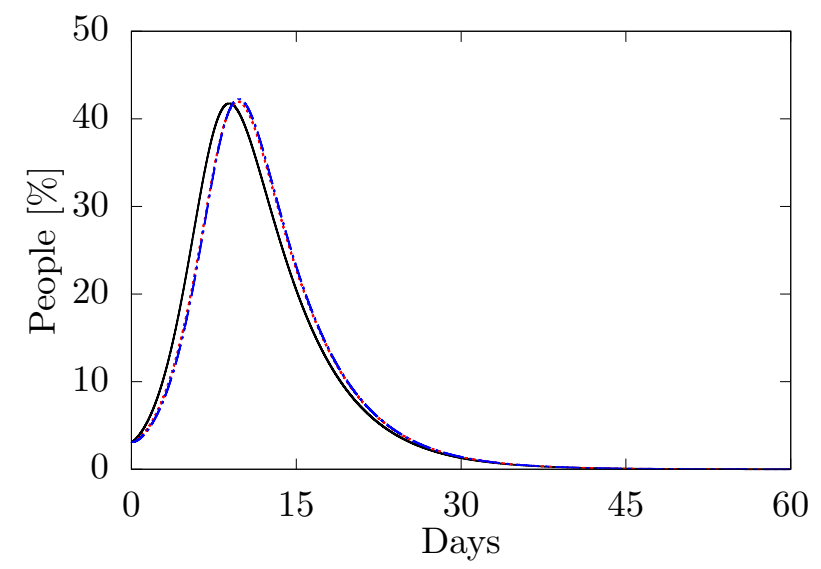

FIG. 4. Time evolution of the fraction of infected people at $d^{\mathcal{I}}=0.001$ and $d^{\mathcal{S}}=d^{\mathcal{R}}=0.0005$ (blue dash-dotted line), 0.001 (red dotted line) and 0.1 (black solid line).

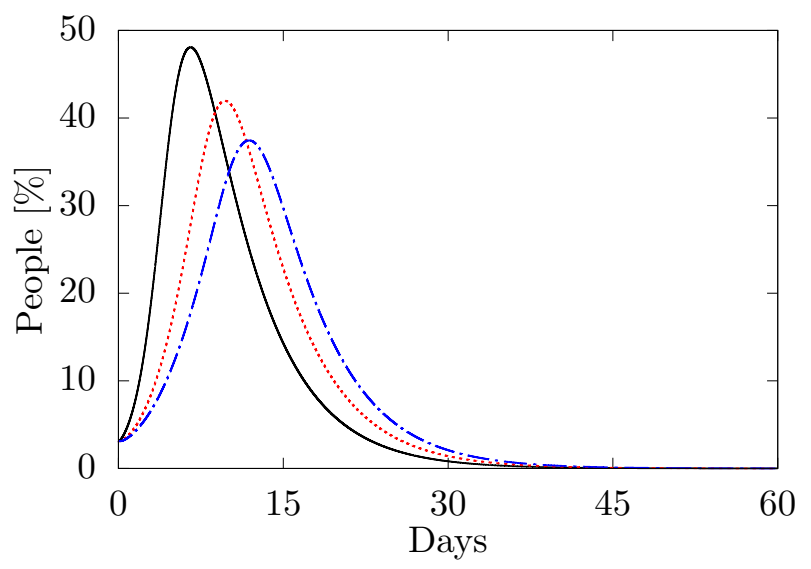

FIG. 5. Time evolution of the fraction of infected people at $d^{\mathcal{S}}=d^{\mathcal{R}}=0.001$ and $d^{\mathcal{I}}=0.0005$ (blue dash-dotted line), 0.001 (red dotted line) and 0.01 (black solid line). 


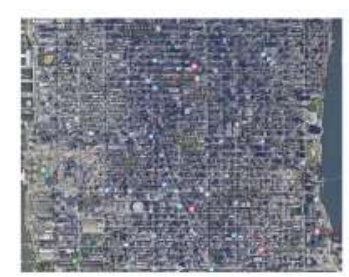

Day 0

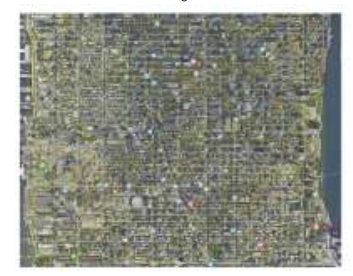

Day 5

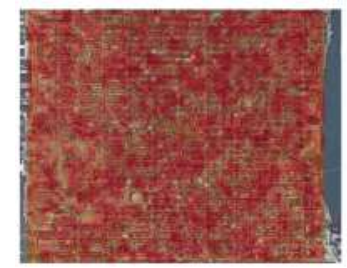

Day 10

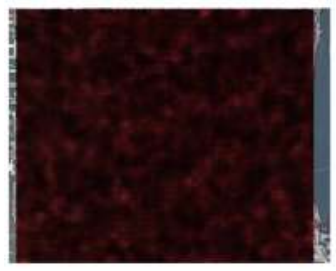

Day 15

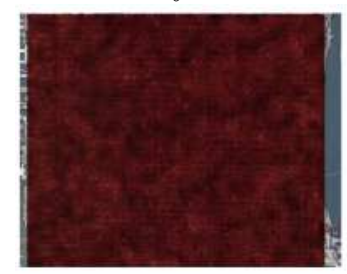

Day 20

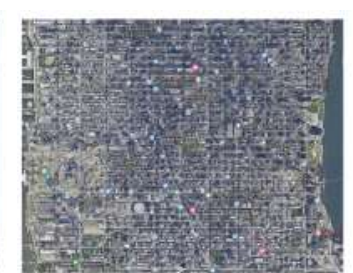

Day 1

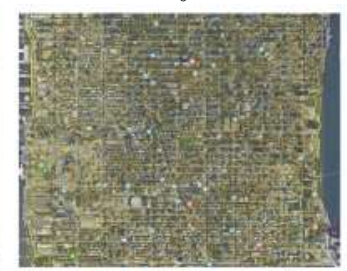

Day 6

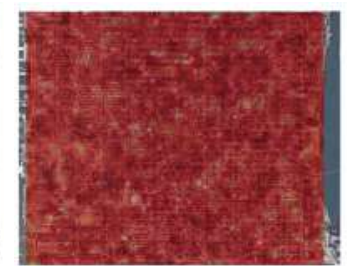

Day 11

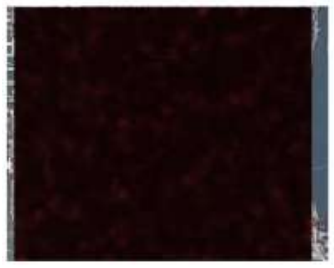

Day 16

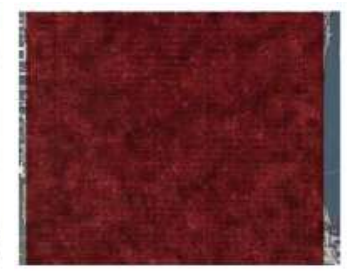

Day 21

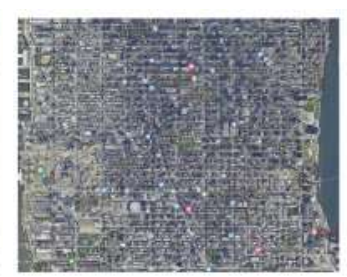

Day 2

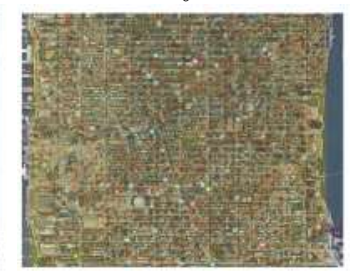

Day 7

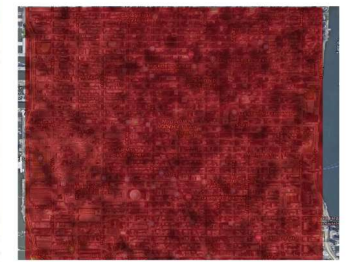

Day 12

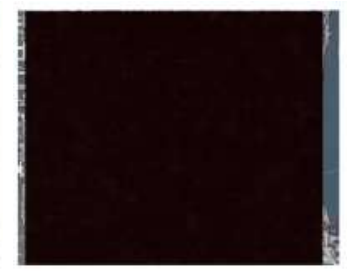

Day 17

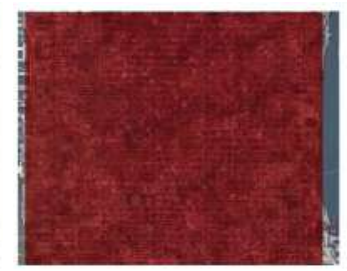

Day 22

0

0.2

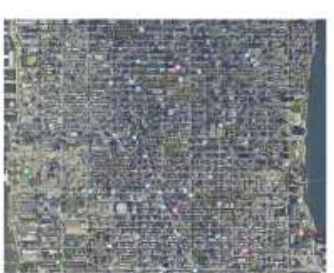

Day 3

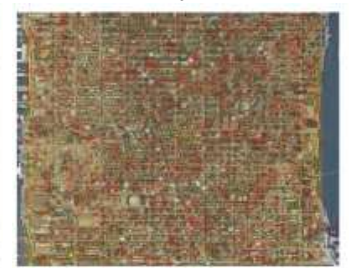

Day 8

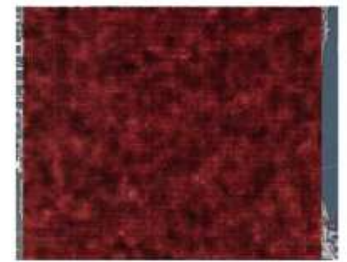

Day 13

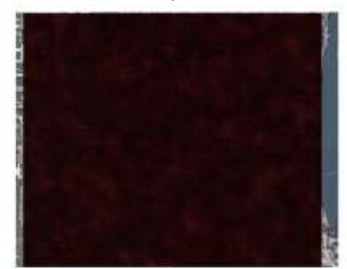

Day 18

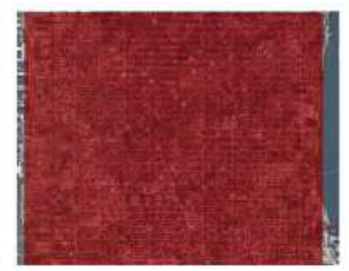

Day 23

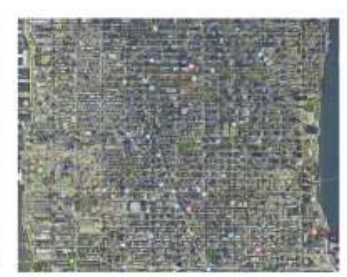

Day 4

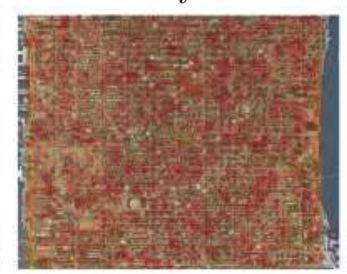

Day 9

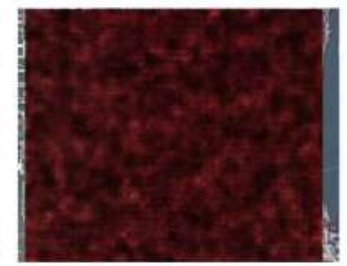

Day 14

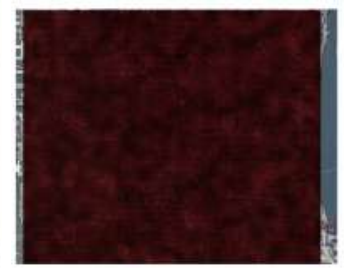

Day 19

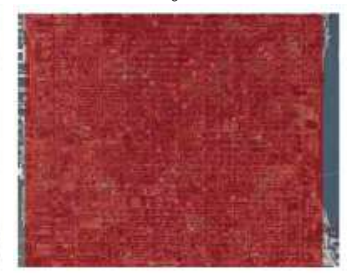

Day 24

FIG. 6. Uniform diffusivity: Map of the percentage fraction of infected people in Midtown Manhattan at different days. 


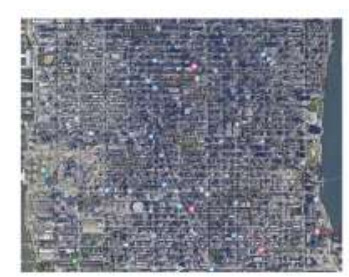

Day 0

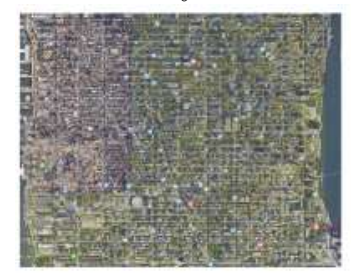

Day 5

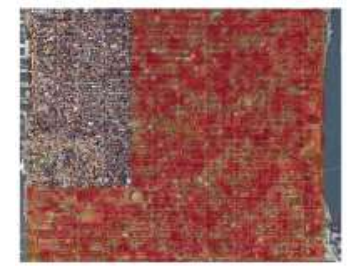

Day 10

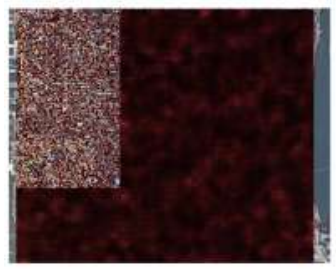

Day 15

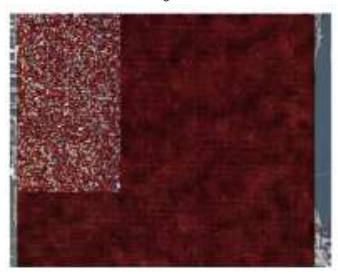

Day 20

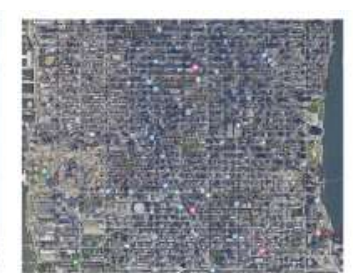

Day 1

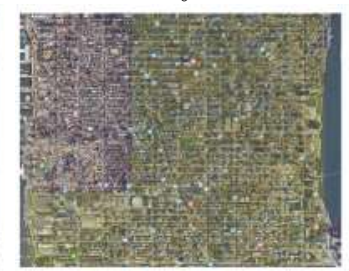

Day 6

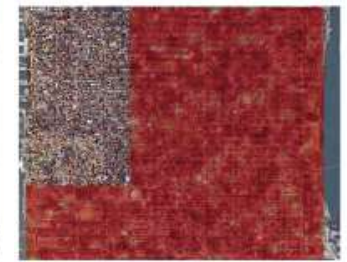

Day 11

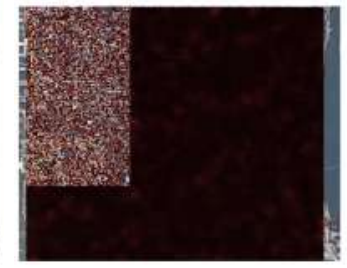

Day 16

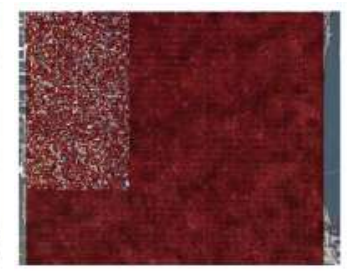

Day 21

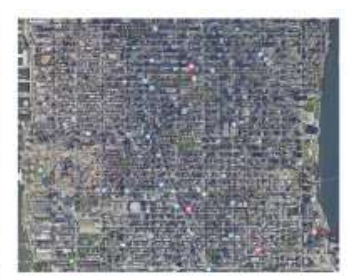

Day 2

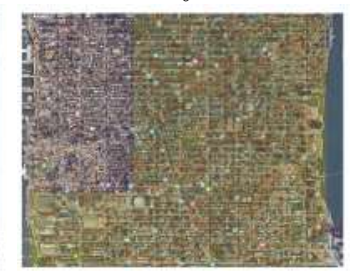

Day 7

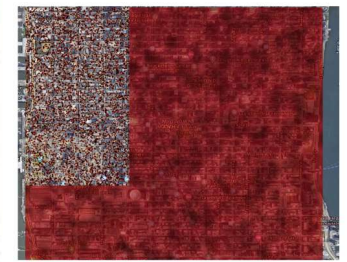

Day 12

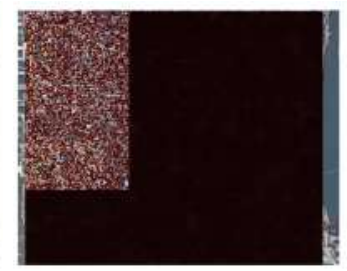

Day 17

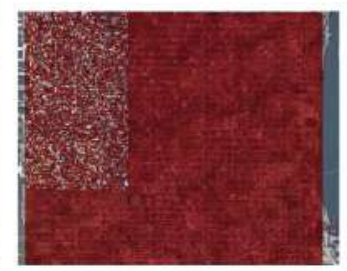

Day 22

0

0.2

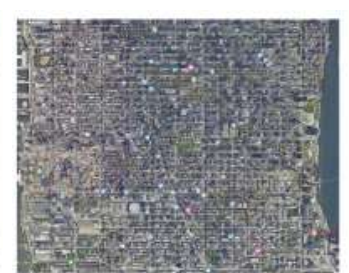

Day 3

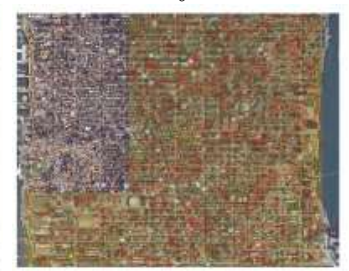

Day 8

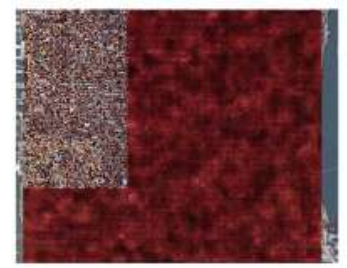

Day 13

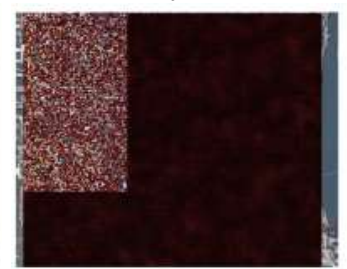

Day 18

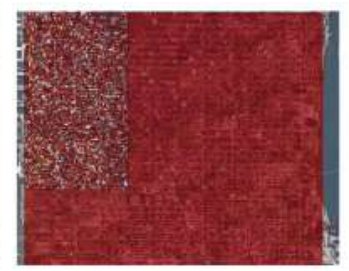

Day 23

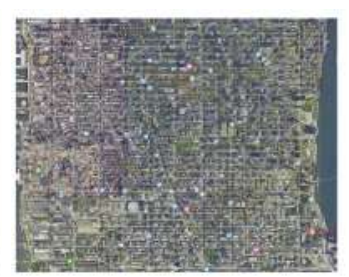

Day 4

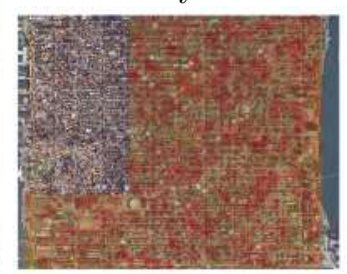

Day 9

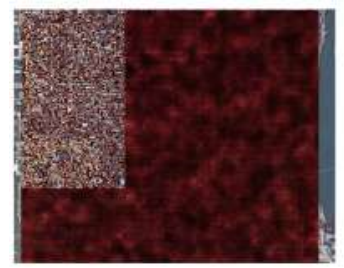

Day 14

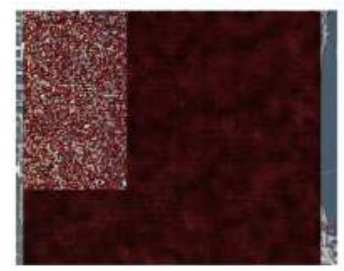

Day 19

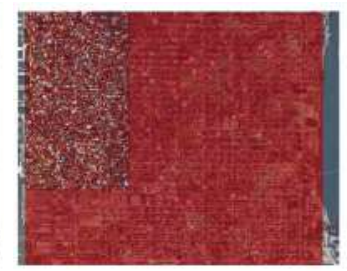

Day 24

FIG. 7. Non-uniform diffusivity: Map of the percentage fraction of infected people in Midtown Manhattan at different days. 


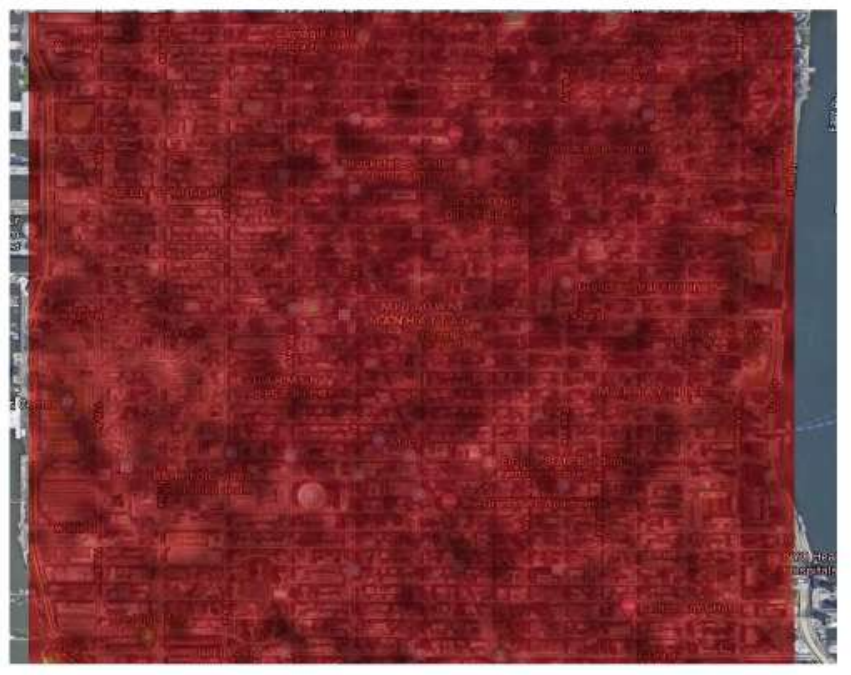

Uniform diffusivity

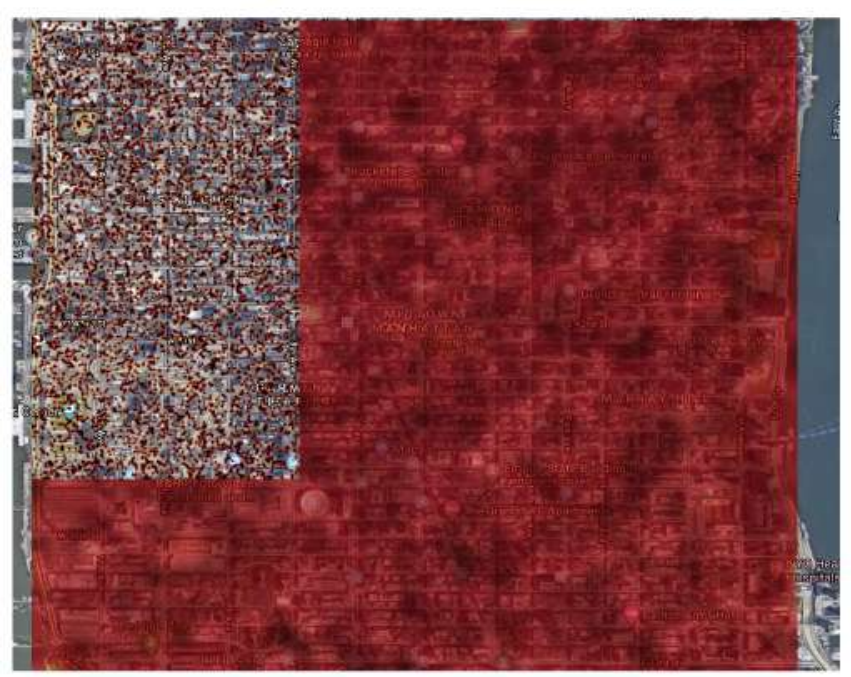

Non-uniform diffusivity

0 0.2

FIG. 8. Uniform vs non-uniform diffusivities: Map of the percentage fraction of infected people in Midtown Manhattan at Day 12.

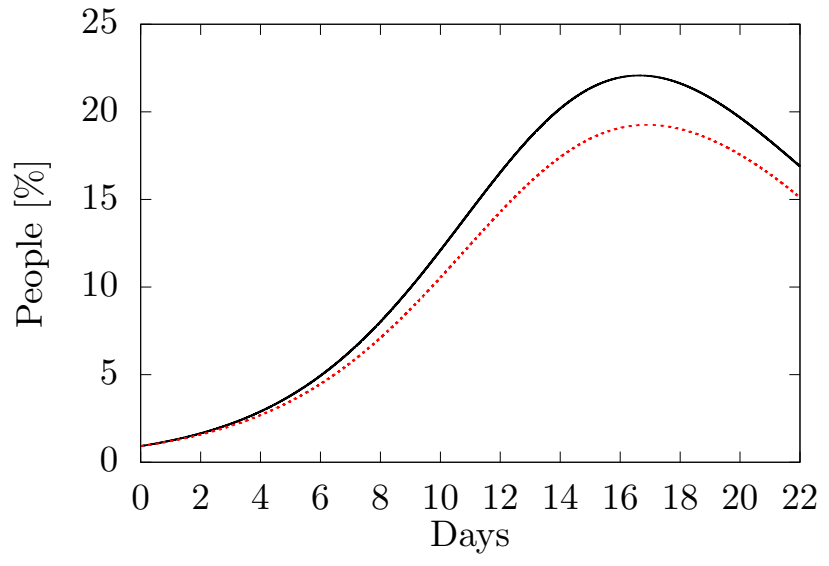

FIG. 9. Time evolution of the fraction of infected people by uniform (black solid line) and non-uniform (red dotted line) diffusivities. 
[1] M. J. Papagrigorakis, C. Yapijakis, P. N. Synodinos, and 369 E. Baziotopoulou-Valavani, International Journal of In-370 fectious Diseases 10, 206 (2006).

[2] D. J. Daley and J. Gani, Epidemic Modelling: An In-372 troduction, Cambridge Studies in Mathematical Biology 373 (Cambridge University Press, 1999).

[3] H. W. Hethcote, SIAM review 42, 599 (2000).

[4] J. Graunt, Ed., WF Willcox. Baltimore (1939).

[5] D. Bernoulli and S. Blower, Reviews in medical virologys 14, 275 (2004).

[6] W. O. Kermack, A. G. McKendrick, and G. T. Walker, 379 Proceedings of the Royal Society of London. Series A,з80 Containing Papers of a Mathematical and Physical Char-381 acter 115, 700 (1927).

[7] D. Mollison, Journal of the Royal Statistical Society: Se-з8з ries B (Methodological) 39, 283 (1977).

[8] P. Elliott and D. Wartenberg, Environmental health per-385 spectives 112, 998 (2004).

[9] A. Turing, Philosophical Transactions of the Royal Soci-387 ety B 237, 37 (1952).

[10] Q.-X. Liu and Z. Jin, Journal of Statistical Mechanics:389 Theory and Experiment 2007, P05002 (2007) -

[11] G. Sun, Z. Jin, Q.-X. Liu, and L. Li, Journal of Statis-391 tical Mechanics: Theory and Experiment 2007, P11011 192 (2007).

[12] G.-Q. Sun, Z. Jin, Q.-X. Liu, and L. Li, Journal of Statis-394 tical Mechanics: Theory and Experiment 2008, P08011 395 (2008).

[13] G.-Q. Sun, Z. Jin, Q.-X. Liu, and L. Li, Journal of Bio-397 logical Systems 17, 141 (2009).

[14] Y. Cai and W. Wang, Journal of Statistical Mechanics:399 Theory and Experiment 2011, P02025 (2011).

[15] W. Wang, Y. Lin, H. Wang, H. Liu, and Y. Tan, Journal ${ }_{401}$ of Biological Systems 19, 19 (2011).

[16] M. Bendahmane and M. Saad, Acta applicandae mathe--403 maticae 115, 17 (2011).

17] W. Wang, Y. Cai, M. Wu, K. Wang, and Z. Li, Nonlinear Analysis: Real World Applications 13, 2240 (2012).

[18] S. Ponce Dawson, S. Chen, and G. D. Doolen, The Jour-407 nal of Chemical Physics 98, 1514 (1993). 408

[19] S. Succi, The Lattice Boltzmann Equation for Fluid Dy-409 namics and Beyond (Clarendon, 2001).

[20] S. Succi, The Lattice Boltzmann Equation: For Complex States of Flowing Matter (Oxford University Press,
2018).

[21] T. Krüger, H. Kusumaatmaja, A. Kuzmin, O. Shardt, G. Silva, and E. M. Viggen, The Lattice Boltzmann Method: Principles and Practice (Springer International Publishing, 2017).

[22] P. Bhatnagar, E. Gross, and M. Krook, Phys. Rev. 94, 511 (1954).

[23] R. Blaak and P. M. Sloot, Computer Physics Communications 129, 256 (2000).

[24] J. Zhang and G. Yan, Journal of Scientific Computing 52, 1 (2012).

[25] J. Latt, Tufts University , 1 (2007).

[26] J. D. Sterling and S. Chen, J. Comput. Phys. 123, 196 (1996).

[27] P. C. Philippi, L. A. Hegele Jr, L. O. Dos Santos, and R. Surmas, Phys. Rev. E 73, 056702 (2006).

[28] D. Siebert, L. Hegele Jr, and P. Philippi, Phys. Rev. E 77, 026707 (2008).

[29] C. Coreixas, G. Wissocq, G. Puigt, J.-F. Boussuge, and P. Sagaut, Phys. Rev. E 96, 033306 (2017).

[30] C. Coreixas, High-order extension of the recursive regularized lattice Boltzmann method, Ph.D. thesis, INP Toulouse (2018).

[31] S. A. Hosseini, C. Coreixas, N. Darabiha, and D. Thévenin, Physical Review E 99, 063305 (2019).

[32] A. De Rosis and K. H. Luo, Phys. Rev. E 99, 013301 (2019).

[33] S. A. Mikheev and G. V. Krivovichev, in Journal of Physics: Conference Series, Vol. 1038 (IOP Publishing, 2018) p. 012040.

[34] G. V. Krivovichev, Applied Mathematics and Computation 348, 25 (2019).

[35] See Supplemental Material at [URL will be inserted by publisher] for four scripts: (i) StabilityAnalysis.m to perform the linear stability analysis, (ii) Units.m to do the conversion from physical units to the LB one, (iii) SIR.m to solve Eqs. (1) and (iv) main.cpp is a $\mathrm{C}++$ program with the implementation of our proposed methodology.

[36] S. Kaushal, A. S. Rajput, S. Bhattacharya, M. Vidyasagar, A. Kumar, M. K. Prakash, and S. Ansumali, arXiv preprint arXiv:2006.00045 (2020). 